\title{
Online Inductance and Capacitance Identification Based on Variable Forgetting Factor Recursive Least-Squares Algorithm for Boost Converter
}

\author{
Chen Chen ${ }^{1}$, Run Min ${ }^{1}$, Qiaoling Tong ${ }^{1}$, Shifei Tao ${ }^{2}$, Dian Lyu ${ }^{1}$, and Linkai Li ${ }^{1}$ \\ ${ }^{1}$ School of Optical and Electronic Information, Huazhong University of Science and Technology, Wuhan 430074, China \\ ${ }^{2}$ Wuhan Marine Machinery Co., Ltd., Wuhan 430074, China
}

\begin{abstract}
The control performance of boost converter suffers from the variations of important component parameters, such as inductance and capacitance. In this paper, an online inductance and capacitance identification based on variable forgetting factor recursive least-squares (VFF-RLS) algorithm for boost converter is proposed. First, accurate inductance and capacitance identification models and the RLS algorithm are introduced. In order to balance the steady-state identification accuracy and parameter tracking ability, a forgetting factor control technique is investigated. By recovering system noise in the error signal of the algorithm, the value of forgetting factor is dynamically calculated. In addition, since the sampling rate is much lower than the existing identification methods, the proposed algorithm is practical for low-cost applications. Finally, the effectiveness of the proposed algorithm is verified by experiment. The experiment results show that the algorithm has good performance in tracking inductance and capacitance variations.
\end{abstract}

\section{Introduction}

Recently, digitally controlled pulsewidth modulated dcdc converters have become a hot research topic because of their lower sensitivity to system noises and component parameter variations, programmability, ability to implement complex control strategies, and possibilities to update controller online [1-5]. The main control techniques of digital dc-dc converter can be classified into voltage mode control and current mode control. The former as a single-loop control can be easily designed and analysed. But it has low response speed to line voltage and load. Compared with the voltage mode control, the current mode control has faster response speed and larger loop gain bandwidth since the inner current loop control is added [6]. The control performance of the two methods depends on accurate converter modeling. As two important parameters in the converter model, the inductance of inductor and capacitance of output capacitor vary over time in practice due to temperature variation, aging effect and load level. Therefore, in order to improve the control performance of dc-dc converter system, it is necessary to identify the inductance and capacitance online for timely tracking their variations.

Several methods for online parameter identification of dc-dc converter have been studied [7-12]. For the load estimation of boost converter, an algebraic parameter identification and classical asymptotic observers are compared in [7]. Different from asymptotic observers, the algebraic identification method does not suffer from stability and convergence issues. Further, Jesús et al. proposes an algebraic identification algorithm for unknown time-varying parameters, such as load resistance and input voltage supply value. On this basis, the generalized proportional-integral based adaptive controller can be tuned online. In [9], a real-time detection technology is studied for capacitance and equivalent resistance (ESR) of output capacitor. With the sampling of capacitor current and ripple voltage, the capacitor parameters can be obtained by Kalman filter. However, the sampling rate is 16 times than the switching frequency, which leads to high requirements for hardware. By analyzing the characteristics of capacitor voltage ripple, a current-sensorless capacitance and ESR identification approach for buck converter is investigated [10]. The output voltage only needs to be sampled twice in a switching cycle. But, the identification accuracy will be affected by inductance variation. In [11], an online identification algorithm for capacitance, ESR, inductance, and load is proposed. Based on the sampling information of MOSFET gate signal, inductor current and output voltage, the algorithm can estimate the above four parameter values through different data processing methods, such as the least mean squares algorithm. In [12], inductance and capacitance can be reconstructed by an observer with adaptive feedback law. For the two methods, since the sampling frequency is much higher than switching frequency and large amounts of data need to be processed, their demands for hardware, such as $\mathrm{ADC}$ and digital signal processor, are high, which is not suitable for low-cost applications. In addition, they are 
difficult to implement in converters with high switching frequency. The above literatures address the online identification of load, input voltage, capacitance, ESR, and inductance. However, none of these algorithms can simultaneously estimate inductance and capacitance with low requirements for system hardware.

In this paper, an inductance and capacitance identification based on variable forgetting factor recursive least-squares (VFF-RLS) algorithm for boost converter is proposed. The value of forgetting factor is derived by recovering system noise in the error signal of the algorithm. Based on the proposed accurate inductance and capacitance identification model, inductance and capacitance variations can be accurately and rapidly tracked. Since the proposed algorithm is simple and its maximum sampling rate is only two times of switching frequency, it is practical for the low-cost applications. The experiment results fully verify the feasibility of the proposed online parameter identification for boost converter.

\section{Online inductance and capacitance identification based on RLS}

The schematic diagram of boost converter with online inductance and capacitance identification algorithm based on RLS is shown in Fig. 1, where $\hat{L}$ and $\hat{C}$ are the identification values of inductance and capacitance, respectively. The digital control of boost converter, illustrated in shaded area, is used to guarantee the stable operation of the system by designing a suitable digital controller. Any kind of constant frequency digital control method can be utilized, such as the classical voltage mode control and current model control.

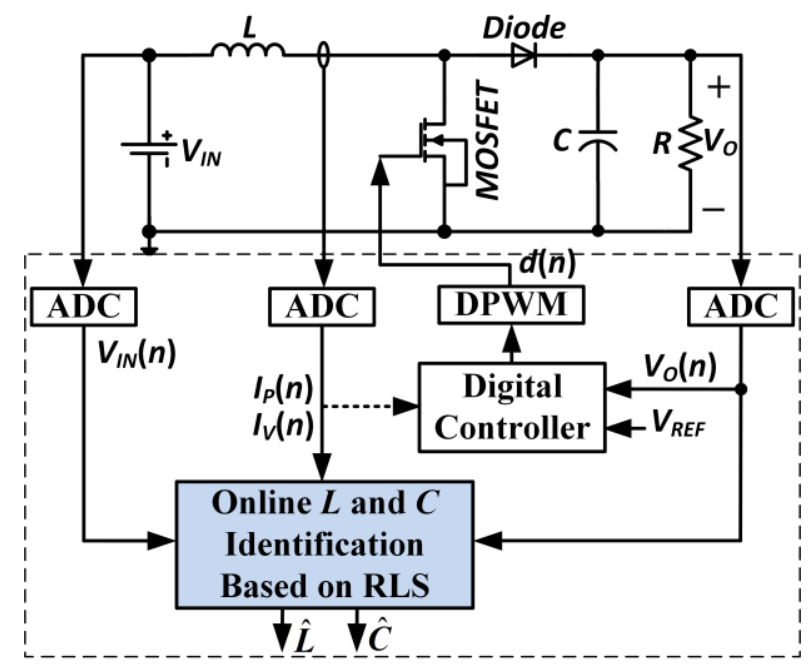

Figure 1. The structure of boost converter with online $L$ and $C$ identification algorithm based on FF-RLS

Since the identification results of inductance and capacitance do not converge in steady state, the identification should be carried out in a dynamic process. First, the dynamic process is created by slightly changing the voltage reference $V_{R E F}$, i.e., a small pulse signal is injected into $V_{R E F}$. Then, according to the sampled data of input voltage, output voltage, and inductor current, inductance and capacitance are identified through the RLS algorithm.

\subsection{Discrete-time modeling of boost converter}

The accuracy of parameter identification is built on the basis of accurate converter modelling. In order to accurately identify the inductance and capacitance, the conventional average state equation of boost converter is first improved.

By considering the inductor current ripple and combining it with the used leading-edge pulse width modulation method, the improved discrete-time average state equation is obtained.

$$
\begin{aligned}
& L \frac{I_{P}(k+1)-I_{P}(k)}{T}=V_{I N}(n)-D^{\prime}(n) V_{O}(n) \\
& C \frac{V_{O}(n+1)-V_{O}(n)}{T}=D^{\prime}(n) \bar{I}_{L}(n) \mid D^{\prime}-\frac{V_{O}(n)}{R}
\end{aligned}
$$

where $V_{O}(n)$ and $I_{P}(n)$ are the sampled output voltage and inductor current at the very beginning of the nth switching cycle, respectively. $\bar{I}_{L}(n) \mid D^{\prime}$ is the average inductor current during the switching off period. It can be expressed as

$$
\bar{I}_{L}(n) \mid D^{\prime}=\frac{I_{P}(n)+I_{V}(n)}{2}
$$

where $I_{V}(n)$ is the valley inductor current of the nth switching cycle, which is sampled in the switch-on moment.

From (1), the inductance and capacitance identification models, which are suitable for the standard RLS method, are given by

$$
\begin{array}{r}
\theta_{L}\left[V_{I N}(n)-D^{\prime}(k) V_{O}(n)\right]=I_{P}(n+1)-I_{P}(n) \\
\theta_{C}\left[D^{\prime}(k) \bar{I}_{L}(n) \mid D^{\prime}-\frac{V_{O}(n)}{R}\right]=V_{O}(n+1)-V_{O}(n)
\end{array}
$$

where $\theta_{L}=T / L, \theta_{C}=T / C$ and they are the parameters to be identified. As can be seen from (3) and (4), the effect of the boost converter's nonlinearity on parameter identification is also eliminated in models. By applying RLS method with (3) and (4), $\theta_{L}$ and $\theta_{C}$ can be calculated, then the inductance and capacitance are estimated.

\subsection{RLS for parameter identification}

Recursive least square algorithm is a widely used adaptive filter. It has the characteristics of fast calculation speed, less calculation, fast convergence and low sensitivity to noise. Therefore, it is commonly applied in the field of parameter identification, especially for online parameter identification.

Recursive formula of RLS algorithm is shown as follows:

$$
\begin{aligned}
e(n) & =y(n)-\hat{\theta}^{T}(n-1) \varphi(n) \\
K(n) & =\frac{P(n-1) \varphi(n)}{\lambda+\varphi^{T}(n) P(n-1) \varphi(n)}
\end{aligned}
$$




$$
\begin{gathered}
\hat{\theta}(n)=\hat{\theta}(n-1)+K(n) e(n) \\
P(n)=\frac{1}{\lambda}\left[I-K(n) \varphi^{T}(n)\right] P(n-1)
\end{gathered}
$$

where $\lambda \in[0,1]$ is the forgetting factor. $e(n), y(n)$, and $\varphi^{T}(n)$ are the error signal, desired output of adaptive filter, and input signal vector, respectively. $\hat{\theta}(n-1)$ is parameter estimation value obtained after $(n-1) t h$ recursion. $K(n) \in R^{N}$ is the Kalman gain, $P(n) \in R^{N \times N}$ is the error covariance matrix, and $N$ is the number of parameters to be identified. The initial value of $P(n)$, i.e., $P(0)$, is selected by the designer with experience.

By applying the RLS method to (3) and (4), respectively, the RLS-based online inductance and capacitance identification is obtained. For inductance identification, $y(n), \varphi(n)$, and $\hat{\theta}(n-1)$ are written as $y(n)=I_{P}(n+1)-I_{P}(n), \varphi(n)=V_{I N}(n)-D^{\prime}(k) V_{O}(n)$, and $\hat{\theta}(n-1)=\hat{\theta}_{L}(n-1)$, while for capacitance identification, $y(n), \varphi(n)$, and $\hat{\theta}(n-1)$ are expressed as $y(n)=V_{O}(n+1)-V_{O}(n), \varphi(n)=D^{\prime}(k) \bar{I}_{L}(n) \mid D^{\prime}-V_{O}(n) / R$ $\hat{\theta}(n-1)=\hat{\theta}_{C}(n-1)$. The identification algorithm is recursively calculated once per switching cycle. According to the duty cycle, the sampled inductor current and voltages, $\varphi(n)$ and $y(n)$ can be obtained. Then Kalman gain $K(n)$ and error signal $e(n)$ is calculated to correct the parameter identification value of last switching cycle, $\hat{\theta}(n-1)$. Thus, the new observation data is constantly introduced in each switching cycle to update the identification value until an expected accuracy is achieved. Finally, the inductance and capacitance can be estimated from the optimal $\hat{\theta}_{L}(n)$ and $\hat{\theta}_{C}(n)$.

\section{VFF-RLS algorithm for inductance and capacitance identification}

In the classical RLS algorithm, $\lambda$ is set to a fixed value. The effect of past observation data is decreased at a fixed rate. If $\lambda$ is close to 1 , the algorithm has high precision and low parameter tracking ability. Decreasing $\lambda$ improves tracking performance, but the steady-state accuracy is also lowered at the same time. In order to satisfy these two contradictory demands, a variable forgetting factor RLS algorithm is investigated for inductance and capacitance identification.

Since the error signal in (5) is a prior error computed with the parameter identification value of the $(n-1) t h$ cycle, it is a prior error. The posteriori error can be expressed as

$$
\varepsilon(n)=y(n)-\hat{\theta}^{T}(n) \varphi(n)
$$

From (5), (7), and (9), $\varepsilon(n)$ can be rewritten as

$$
\varepsilon(n)=e(n)\left[1-\varphi^{T}(n) K(n)\right]
$$

The value of forgetting factor is designed by recovering the system noise in the error signal of the adaptive filter, i.e., the forgetting factor $\lambda(n)$ can be adjusted according to (11).

$$
E\{e(n) \varepsilon(n)\}=E\left\{v^{2}(n)\right\}
$$

where $E\left\{v^{2}(n)\right\}=\sigma_{v}^{2}$ is the power of system noise.

Substituting (6) and (10) into (11), with the assumption that the input and error signals are uncorrelated, (12) can be obtained.

$$
E\left\{\frac{\lambda(n)}{\lambda(n)+q(n)}\right\}=\frac{\sigma_{v}^{2}}{\sigma_{e}^{2}(n)}
$$

where $q(n)=\varphi^{T}(n) P(n-1) \varphi(n), E\left\{e^{2}(n)\right\}=\sigma_{e}^{2}(n)$ is the power of a priori error signal. The assumption is true when the identified parameters have started to converge to the actual value. By solving (12), the variable forgetting factor is given by

$$
\lambda(n)=\frac{\sigma_{v}^{2} \sigma_{q}^{2}(n)}{\sigma_{e}^{2}(n)-\sigma_{v}^{2}}
$$

where $E\left\{q^{2}(n)\right\}=\sigma_{q}^{2}(n)$. In real application, $\sigma_{e}^{2}(n)$ and $\sigma_{q}^{2}(n)$ can be estimated by

$$
\begin{aligned}
& \hat{\sigma}_{e}^{2}(n)=\alpha \hat{\sigma}_{e}^{2}(n-1)+(1-\alpha) e^{2}(n) \\
& \hat{\sigma}_{q}^{2}(n)=\alpha \hat{\sigma}_{q}^{2}(n-1)+(1-\alpha) q^{2}(n)
\end{aligned}
$$

where $\alpha \in[0,1]$ is the weighting factor.

Since the value of $\lambda$ must be in the range of $[0,1]$, the forgetting factor of the proposed algorithm is given by

$$
\lambda(n)=\min \left\{\frac{\sigma_{v}^{2} \hat{\sigma}_{q}^{2}(n)}{\xi+\hat{\sigma}_{e}^{2}(n)-\sigma_{v}^{2}}, \lambda_{\max }\right\}
$$

where $\xi$ is a small positive constant that prevents the division by zero. Before the identification results converge or when an abrupt change occurs in the system, $\hat{\sigma}_{e}^{2}(n)$ is larger than $\sigma_{v}^{2}$, hence $\lambda(n)$ goes to lower values, which makes the algorithm achieves fast convergence and tracking. When the results converge to the steady-state value, $\lambda(n)$ is set to $\lambda_{\max }$, then the algorithm can have high identification accuracy.

\section{Experiment results}

To validate the performance of the proposed identification method, a prototype is employed for testing the steady-state accuracy and tracking ability of the proposed identification. Experimental system hardware consists of a control stage and power stage. The core component of control stage is Texas Instruments TMS320F2812 DSP system, which is used to implement the control and parameter identification algorithms. The power stage contains a non-synchronous boost converter and signal conversion circuits. The specifications of the boost converter are shown in Table 1. The nominal inductance is obtained by connecting two inductors $\left(L_{1}=28 \mu \mathrm{H}\right.$ and $\left.L_{2}=100 \mu \mathrm{H}\right)$ in parallel. Similarly, the nominal capacitance is got by connecting two capacitors $\left(C_{1}=56 \mu \mathrm{F}\right.$ and $\left.C_{2}=10 \mu \mathrm{F}\right)$ in series. The digital peak current control is utilized to ensure the system stable operation. 
Table 1. Specifications of the tested boost converter.

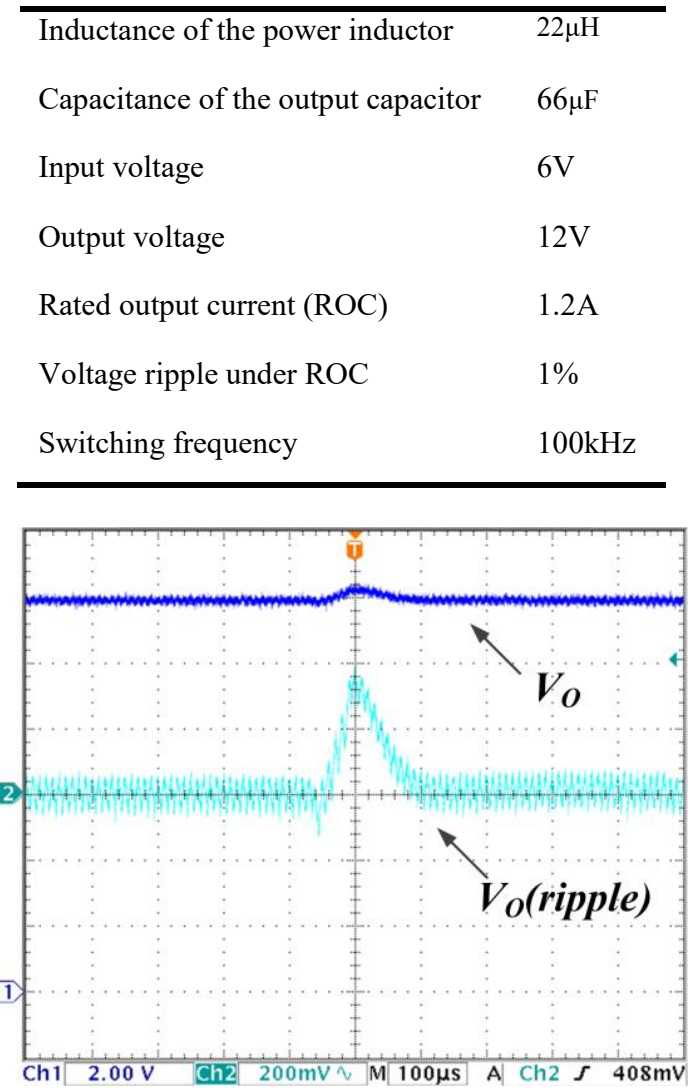

Figure 2. Output voltage waveform when small pulse signal is injected

Fig. 2 shows the output voltage waveform during the small pulse injection. The injected pulse signal lasted $20 \mu \mathrm{s}$. Its amplitude is $3 \%$ of expected voltage reference. As can be seen from Fig. 2, after the voltage reference changes, there is a transient response in the output voltage. With the recovery of voltage reference, the output voltage then returns to the steady-state. The whole process lasts $200 \mu \mathrm{s}$. The maximum derivation of output voltage is $0.32 \mathrm{~V}$, which is less than $3 \%$ of the steadystate output voltage. Therefore, the injection effect on output voltage is small. The boost converter can be regarded as always working in steady state.

During the transient state, the input voltage, output voltage, and inductor current are sampled by $\mathrm{AD}$ converters, providing the data for estimating inductance and capacitance. In order to verify the robustness of the proposed algorithm, the parameter identification is executed with different input voltages and the initial values of inductance and capacitance are set to $20 \mu \mathrm{H}$ and $56 \mu \mathrm{H}$, which derivate from the actual values. The identification results are shown in Fig. 3 and Fig. 4, respectively. As Fig. 3 shows, under the rated input voltage, the identification result of inductance converges to $20.95 \mu \mathrm{H}$ after 14 iterations. The identification error is only $4.77 \%$ compared with the actual value $(22 \mu \mathrm{H})$. When the input voltage decreased to $5 \mathrm{~V}$, the identified inductance is $21.07 \mu \mathrm{H}$. The estimated error is still low. As can be seen from Fig. 4, under the rated input voltage, the identification result of capacitance converges to $67.64 \mu \mathrm{F}$ after 14 iterations with $2.48 \%$ estimation error.
When the input voltage is $5 \mathrm{~V}$, the estimated capacitance is $67.69 \mu \mathrm{F}$, of which the estimation error is still very small. Therefore, the proposed algorithm can accurately identify the inductance and capacitance and will not affected by changes in input voltage.

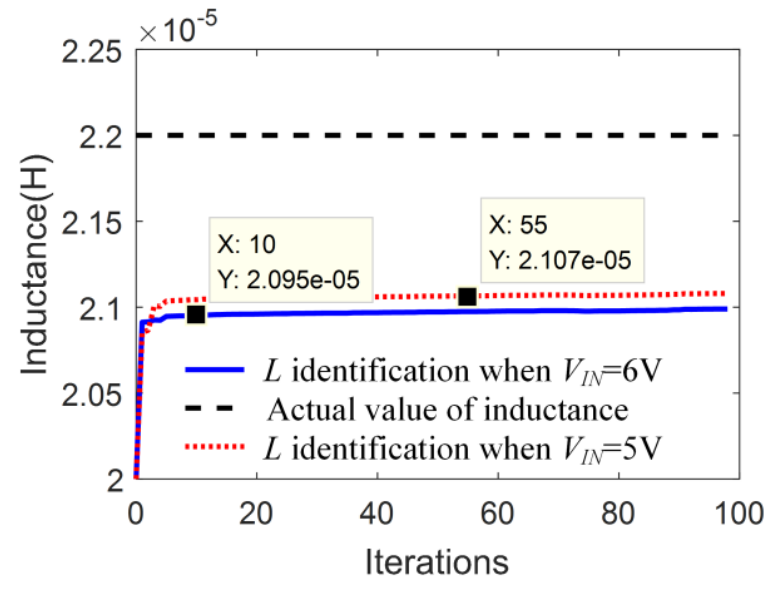

Figure 3. Identification results of inductance under different input voltages

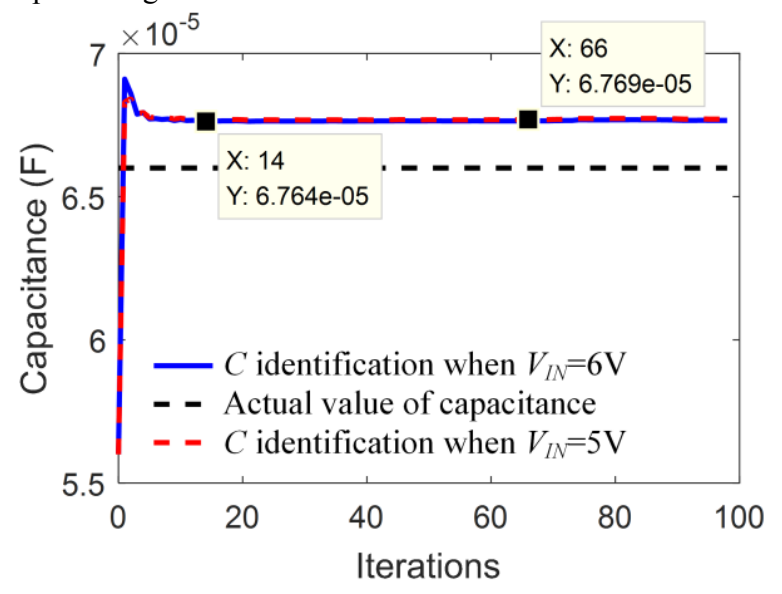

Figure 4. Identification results of capacitance under different input voltages

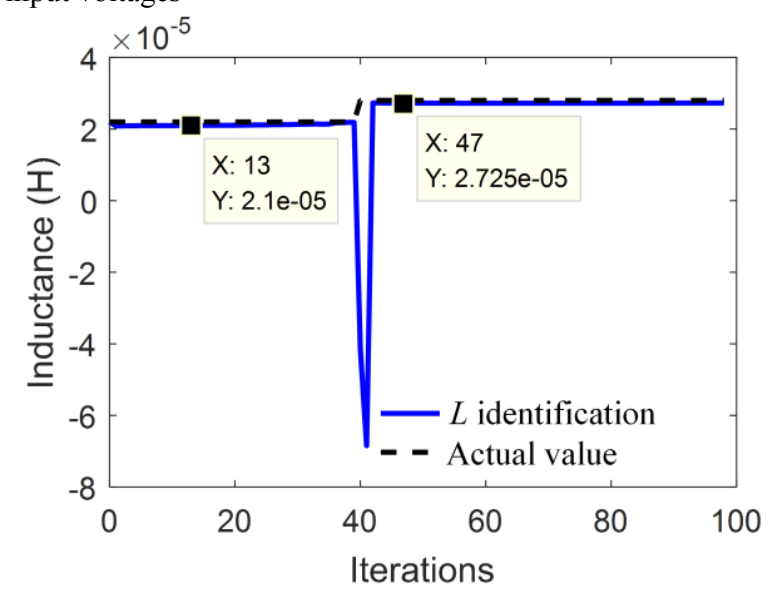

Figure 5. Identification results of inductance under inductance and capacitance variations

In order to verify the tracking capabilities and steady state accuracy of the proposed algorithm, step changes in inductance and capacitance are applied. To simulate a sudden change in inductance and capacitance, the inductor $L_{2}$ and capacitor $C_{2}$ are simultaneously and transiently disconneted from the boost converter. The 
identification results are shown in Fig. 5 and Fig. 6, respectively. As can be seen from Fig. 5 and Fig. 6, when inductance and capacitance step change, the estimated inductance and capacitance converge to the steady-state after 7 and 10 iterations. The identification results are $27.25 \mu \mathrm{H}$ and $56.83 \mu \mathrm{F}$, respectively, of which the estimation errors are less than $2.7 \%$.

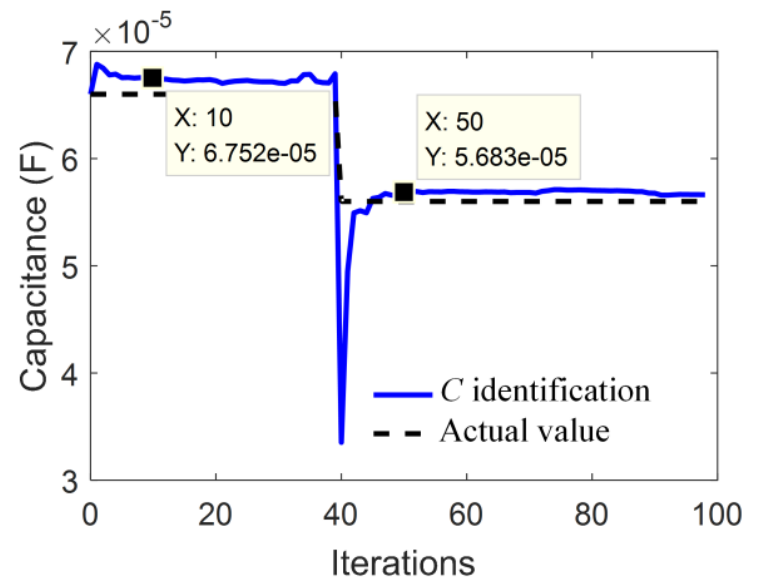

Figure 6. Identification results of capacitance under inductance and capacitance variations

As can be seen from the experiment results, even if there are sudden changes in inductance and capacitance, the errors between the identified values and actual values are always less than $5 \%$. The proposed algorithm can track the actual inductance and capacitance quickly and precisely.

\section{Conclusion}

An online inductance and capacitance identification based on VFF-RLS algorithm is proposed in this paper. With the consideration of inductor current ripple, the inductance and capacitance identification models for standard RLS method are derived. By recovering the system noise in the error signal of the algorithm, the value of forgetting factor in RLS algorithm is dynamically calculated, which solves the problem of the classical RLS algorithm that cannot balance the steadystate accuracy and parameter tracking ability. The experiment results indicate that the proposed algorithm can accurately and rapidly track the inductance and capacitance variations. In addition, the algorithm is simple and easy to implement in practice, which is suitable for low-cost applications. Since the proposed algorithm is applied for boost converters, a further investigation of the application on other topologies and the effect of parasitic parameters on converter modeling, still need to be considered in future works.

\section{Acknowledgement}

The financial support of this research by the National Science Foundation of China, under Grant No. 61202469, 61640311 is greatly appreciated.

\section{References}

1. G. Feng, E. Meyer, Y.F. Liu. A new digital control algorithm to achieve optimal dynamic performance in DC-to-DC converters. IEEE Trans. Power. Electron., 22, 4 (2007)

2. Y.S. Lai and C.A. Yeh, Predictive digital-controlled converter with peak current-mode control and leading-edge modulation. IEEE Trans. Ind. Electron., 56, 6 (2009)

3. M. Veerachary and R. Saxena, Design of robust digital stabilizing controller for fourth-order boost DC-DC converter: A quantitative feedback theory approach, IEEE Trans. Ind. Electron., 59, 2 (2012)

4. Q. Tong, Q. Zhang, R. Min, X. Zou, Z. Liu, and Z. Chen. Sensorless predictive peak current control for boost converter using comprehensive compensation strategy. IEEE Trans. Ind. Electron., 61, 6 (2014)

5. Y. Qiu, H. Liu, and X. Chen, Digital average currentmode control of PWM DC-DC converters without current sensors. IEEE Trans. Ind. Electron., 57, 5 (2010)

6. Q. Zhang, R. Min, Q. Tong, X. Zou, Z. Liu, and A. Shen. Sensorless predictive current controller DCDC converter with a self-correction differential current observer. IEEE Trans. Ind. Electron., 61, 12 (2014)

7. Gensior A, Weber J, Rudolph J, and H. Güldner. Algebraic Parameter Identification and Asymptotic Estimation of the Load of a Boost Converter. IEEE Trans. Ind. Electron., 55, 9 (2008)

8. L.F. Jesús, H.M. Arturo, G.R. Carlos, and H.S. Ramírez. Robust nonlinear adaptive control of a "boost" converter via algebraic parameter identification. IEEE Trans. Ind. Electron., 61, 8 (2014)

9. K. Abdennadher, P. Venet, G. Rojat, J.M. Rétif, and C. Rosset. A real-time predictive-maintenance system of aluminum electrolytic capacitors used in uninterrupted power supplies. IEEE Trans. Ind. Appl., 46, 4 (2010)

10. K. Yao,W. Tang,W. Hu, and J. Lyu, A currentsensorless online ESR and C identification method for output capacitor of buck converter. IEEE Trans. Power Electron., 30, 12 (2015)

11. G. Buiatti, A. Amaral, and A. Cardoso, An online technique for estimating the parameters of passive components in non-isolated DC/DC converters. In Proc. IEEE Int. Symp. Ind. Electron., 606-610 (2007)

12. Z. Cen and P. Stewart, Condition parameter estimation for photovoltaic buck converters based on adaptive model observers. IEEE Trans. Rel., 66, 1 (2017) 